\title{
EL INNATISMO Y LA CIENCIA: UNA HISTORIA DE NAUFRAGIOS Y UNA ISLA DESIERTA
}

\author{
NATIVISM AND SCIENCE: A HISTORY OF \\ SHIPWRECKS AND A DESERTED ISLAND
}

\author{
Guillermo LORENZO \\ Universidad de Oviedo*
}

Resumen: La teoría clásica de las ideas innatas conoció en las últimas décadas un resurgir de la mano de filosofía chomskyana del lenguaje y la mente al amparo de una coartada genética. Sin embargo, la clarificación del alcance del papel de los genes en el desarrollo y de la complejidad y pluralidad de los sistemas en que aquellos se integran ha ensombrecido, si no apagado definitivamente, la infuencia del planteamiento. En este trabajo se argumenta, además, que históricamente se produjo un efecto semejante con el innatismo cartesiano, al ser interpretado como un modelo disposicional de lo innato y acomodado al modelo epigeneticista del desarrollo orgánico. El precio de la supervivencia de alguna forma de innatismo parece ser el de permanecer aislado de cualquier lectura naturalista o científica y reafirmarse como una postura metafísica, epítome de la cual sería el modelo monadológico de Leibniz.

\footnotetext{
* Profesor Titular de Universidad. Facultad de Filosofía y Letras, Campus de Humanidades, E-33011 Oviedo. glorenzo@uniovi.es. Financiación: Desarrollo, Adquisición y Mecanismos de Variación Lingüística (DALiV), FFI2017-87699-P. Este texto está dedicado a la memoria de Eloy Rada. Agradezco al revisor anónimo sus relevantes comentarios. Los errores que puedan permanecer en el texto son de mi entera responsabilidad.
} 
Palabras clave: Innatismo, Naturalismo, Chomsky, Descartes, Liebniz.

Abstract: The classic theory of innate ideas experienced a resurge in the last decades through the Chomskyan philosophy of mind and language and in association with a genetic justification. However, the clarification of the role and scope of the genes in development, along with the complexity and plurality of the systems that comprise them has shadowed, perhaps even blown out, the reputation of this stance. Historically, it is argued here that a similar effect happened regarding the Cartesian nativism, when interpreted as a dispositional model of the innate and accommodated to the epigeneticist model of development. The price of the survival of some kind of nativism appears to be to maintain itself isolated from any naturalistic reading and to stress its metaphysical character, the epitome of which is Leibniz's monadological model.

KeYwords: Nativism, Naturalism, Chomsky, Descartes, Leibniz.

La idea de "innatismo" nunca ha sido clara ni ha tenido un contenido unánime entre sus defensores (Cowie, 1999; Lorenzo y Longa, 2018; Samet, 2008). Tampoco ha dado nunca lugar a un concepto científico de lo "innato", ni en el pasado ni en tiempos recientes, lo que no significa que no haya habido empeños en tal dirección (Mehler y Dupoux, 1990). Según los casos y las interpretaciones a que han dado lugar, la tesis innatista ha servido alternativamente para nombrar una negación (la de que todos los contenidos de una mente sean derivables de la experiencia), una dejación (la de ofrecer una explicación naturalista a reductos especialmente resistentes de la cognición) o una conexión metafísica (la existente entre la divinidad y la mente individual). A partir de ninguna de estas tres vertientes de la posición innatista es factible, de hecho, derivar ningún tipo de principio genuinamente explicativo sobre la localización en la mente de ciertas ideas o de los mecanismos subyacentes a ellas.

Ya a principios del siglo XX, el psicobiólogo Zing Yang Kuo opuso una aguda crítica al innatismo de los instintivistas norteamericanos de la época, basada en que la idea de "instinto", como en general la de "innato", era solo la marca de una psicología "acabada", en el sentido de "acabada a destiempo", ya que ofrecía como explicación aquello que realmente debería recibirla (Kuo, 1921; 1922; 1924; para una síntesis, Kuo, 1967). En semejante línea de ataque basó también Daniel Lehrman la suya al instintivismo, mucho más sofisticado, de Konrad Lorenz. De acuerdo con Lehrman (1953), la apelación a instintos supone la asunción implícita de un (pseudo) principio metodológico de 
renuncia a investigar el valor formativo del desarrollo, comenzando por la historia prenatal del organismo. Por citar un solo ejemplo, pero muy representativo e históricamente relevante, la postulación de un instinto de picoteo en muchas especies de aves, adaptativamente adecuado a las necesidades nutritivas de los polluelos, fue defendido ya, por ejemplo, por Pierre-Jean Cabanis, uno de los más célebres instintivistas pre-darwinianos (Richards, 1987). Sin embargo, Zing Yang Kuo pudo demostrar experimentalmente que no se trata sino de un hábito derivado y continuador del cabeceo fetal debido a los espasmos del corazón sobre el endeble cuerpo en formación (Kuo, 1932). A ejemplos como este se sumarían posteriormente muchos más, comenzando por los planteados en los definitivos trabajos de Gilbert Gottlieb sobre el papel determinante de la experiencia prenatal en el desarrollo y modulación del canto de buen número de aves (para una síntesis, Gottlieb, 1997). Este tipo de críticas, respaldadas además por brillantes estudios experimentales en muchos casos, apuntalan la idea de que la posición innatista no ha supuesto, con frecuencia, otra cosa que la renuncia a prolongar la investigación más allá de lo obvio.

Más cercanas en el tiempo, las teorías chomskianas sobre el lenguaje y la mente han supuesto un reverdecer de la posición innatista, si bien no debidamente justificado (para una visión de conjunto de aquellas, véase Lorenzo 2001). Chomsky, por un lado, ciñéndose al modelo clásico de los argumentos de pobreza del estímulo, basa el innatismo de las categorías y principios computacionales básicos del lenguaje en la infrarrepresentación en los datos (es decir, las emisiones lingüísticas exteriorizadas) de señales indicativas de los mismos, lo que hace inviable que puedan llegar a instalarse en la mente infantil mediante aprendizaje (Berwick et al., 2011; Chomsky, 1967; 2016). Esto implica que, para Chomsky, "innato" equivale a "no aprendido" y que es, por tanto, un concepto sin contenido o aportación positiva propia (Longa y Lorenzo, 2012).

Chomsky ha intentado corregir esta carencia remitiendo las categorías y principios en cuestión, total o parcialmente, al genotipo humano (Chomsky, 1980; 2005). Sin embargo, esta estrategia correctiva no encuentra fundamento alguno en la genética. Por dos razones, al menos. En primer lugar, porque los genes no son "acerca de" nada, es decir, no son entidades intencionales que puedan estar en lugar de esto o aquello (órganos, ideas, hábitos, etc.; tampoco, claro, categorías o principios computacionales) (Balari, Longa y Lorenzo, 2020; Griffiths y Stotz, 2013; Longa, 2018). En segundo lugar, porque la implicación causal de los genes en el desarrollo de las estructuras neurales a cargo de esas categorías y principios, aunque plausible, no puede ser más que una pequeña parte de complejos sistemas 
de desarrollo en que nada en particular tiene un valor explicativo como el que se le supone por sí sola a la contribución genética (Balari, Longa y Lorenzo, 2020; Longa y Lorenzo, 2012). Esto nos devuelve, de nuevo, a las críticas de los psicobiólogos del desarrollo a los modelos instintivistas clásicos (aplaudidos estos, por cierto, repetidamente por Chomsky; Chomsky, 1959; 2009; 2012): el innatismo chomskiano implica también la renuncia a explorar el valor formativo de la mayor parte de los innumerables recursos y procesos, tanto internos al organismo como ambientales, que concurren en la larga historia de desarrollo del niño prelingüístico (Balari, Lorenzo y Sultan, 2020; Locke, 1983; Longa, 2008). La genética, pues, no ha satisfecho la promesa de cobijo que el innatismo esperaba encontrar en ella.

Los perfiles de la aspiración innatista a dotarse de crédito científico podrían, en principio, haber sido diferentes en el caso de la reivindicación de las ideas innatas por parte del racionalismo histórico. Para el racionalista clásico, la postulación de ideas innatas localizadas en la mente individual no va (necesariamente) acompañada de la aceptación del desafío de su eventual refutación por la vía empírica. En una lógica que en cierto modo funciona en el sentido inverso a la del descubrimiento científico, el racionalista basa en la inevitabilidad del innatismo de, al menos, algunas ideas la participación de la mente individual en una mente trascendente a ella: en otras palabras, la irrefutabilidad, no empírica, sino metafísica (en todo caso comparable al modelo de las ciencias exactas; Peńa, 2005), de la conexión entre lo divino y lo humano. Con este trasfondo, resulta particularmente llamativa la propensión del cartesianismo a acomodar la tesis innatista a un formato científicamente tolerable (Longa y Lorenzo, 2018, capítulo 3).

En todo caso, si Descartes siguió ese camino no fue directamente por la ambición de establecer una posición innatista científicamente incontestable. Más bien, la prefiguración de un innatismo científicamente acomodable fue un efecto lateral de su respuesta a la crítica de que la mente requiriese el uso de la razón para obtener el conocimiento, o mayor claridad en el conocimiento, de parte del caudal de ideas innatas accesibles al hombre. En esta crítica al innatismo racionalista incidió posteriormente, por cierto, Locke en 1690 (Ensayo...), pero Descartes ya había tenido que enfrentarse a ella en 1648 (Observaciones...). En la formulación de Locke, la crítica se asienta en la contradicción que supone que la mente conozca de antemano y tenga que obtener, sin embargo, el conocimiento de ciertas ideas: es tanto como afirmar que la mente las conoce y no las conoce al mismo tiempo. La posición de Descartes, que en tiempos más recientes ha 
recibido el nombre de "innatismo disposicional" (Kim, 2003; Rawson, 2006; Stich, 1975), es que el concepto de "idea innata" no presupone la "preexistencia" de este tipo de idea. Las ideas no son innatas en ese sentido. Lo genuinamente innato es la capacidad para pensarlas, definitoria de lo humano como cualquier otro de los atributos orgánicos típicos de la especie (Smit, 2020). Las ideas son innatas por su accesibilidad a esta capacidad de pensamiento y, entre aquellas, algunas no requieren más que la actuación de tal capacidad para instalarse en la mente, sin necesidad de contribución alguna de la experiencia. Este innatismo disposicional tiene el efecto, por un lado, de transformar en innatas todas las ideas, en la medida en que implican sin excepción la facultad humana innata de pensarlas (Gorham, 2002). Tiene, por otro lado, el efecto de equiparar la capacidad o facultad innata de pensar con cualquier otro atributo orgánico innato de la especie, lo que sirve, además, para explicar que no todas las mentes de hecho actualicen las mismas ideas. Como cualquier otro rasgo de especie, la capacidad de pensar está sujeta a variación individual en su desarrollo y en las contingencias que en cada caso hayan podido motivar su uso. El innatismo disposicional cartesiano queda ejemplarmente retratado en el siguiente fragmento:

En realidad nunca he escrito que la mente precise de ideas innatas, que fuesen algo diverso de su propia facultad de pensar. Más bien, advirtiendo la existencia en mí de algunos pensamientos que no procedían de los objetos externos ni de la determinación de mi voluntad sino de la facultad de pensar que poseo, con el fin de distinguir las ideas o nociones que son las formas de estos actos de pensar de aquellas otras que son adventicias o construidas, he decidido llamar a las primeras innatas. Uso este término en el mismo sentido que cuando afirmamos que la generosidad es innata en algunas familias y que en otras lo son algunas enfermedades como la gota o el cálculo, pero no en el sentido de que los hijos de esas familias padezcan estas enfermedades desde el vientre de sus madres, sino en el sentido de que nacen con cierta disposición o facultad para adquirirlas (Descartes, 1648/1981, 20-21).

En este contexto, resulta importante resaltar que Descartes fue un abierto defensor de las tesis epigeneticistas (Aucante, 2006), según las cuales los diferentes atributos de un organismo son efecto de su desarrollo; en otras palabras, dichos atributos no existen desde un primer momento en algo así como una 
versión mínima e inmadura de sí mismos, para después desplegarse hasta alcanzar su forma y madurez finales, como en cambio defendía el preformacionismo. En la época, el epigeneticismo contaba con el favor de la mayor parte de los filósofos y con el placet de la Iglesia, tal vez por el peso del venerable antecedente de Aristóteles, que también lo abrazó (Maienschein, 2012). No debe sorprender, pues, que Descartes lo defendiese y aplicase, ejemplarmente, en su Tratado del feto (1637). Allí, escribió que el estado prístino de cualquier animal es una semilla que lejos de contener una miniatura de sí mismo,

parece no ser más que una mezcla confusa de dos líquidos que, sirviendo de levadura uno al otro, se recalientan de modo que algunas de sus partículas adquieren la misma agitación que en el fuego, por lo que a la vez se dilatan y toman a las otras y por este medio las disponen poco a poco de la forma requerida para formar los miembros (Descartes 1637/1987, 106).

A partir de estos "confusos" orígenes, Descartes describe minuciosamente un sucederse de colisiones, compresiones o rarefacciones que van generando las formas que a su vez van constriñendo el curso de sucesivos procesos semejantes, empezando por el corazón, seguido por el cerebro, etc. Sin excepción, todos los procesos y constricciones apelados son estrictamente mecánicos: filtros y obstáculos físicos interpuestos a las partículas orgánicas originarias, que van dando sucesivamente lugar a la implantación de órganos precursores, a su duplicación o a su diversificación y especialización definitivas. Al defender, a su vez, la tesis disposicional, que convierte el pensamiento en una especie de dispositivo orgánico innato para la generación de las ideas accesibles a la mente individual, y al romper la ecuación entre "innato" y "preexistente" (o "preformado"), se sigue que, implícitamente al menos, Descartes ofrecía al innatismo de las ideas la posibilidad de desarrollarse como teoría científica al abrigo de las tesis epigeneticistas sobre el crecimiento orgánico.

La tesis disposicional sobre el innatismo de las ideas fue asimismo abrazada por Leibniz en su primera aproximación a la materia, como respuesta explícita a los ataques de Locke, en la primera parte de los Nuevos ensayos sobre el entendimiento humano (1704) (Savile, 1972). Allí defiende que las más complejas o abstractas de las ideas son accesibles a cualquier mente sin el apoyo de 
otras aprendidas "por medio de la experiencia o por tradición ajena" (Leibniz, $1704 / 1992,74)$. Son innatas las ideas para cuyo descubrimiento existe una "disposición" (Leibniz, 1704/1992, 78) mental y de ellas puede decirse que son conocidas por todos, aunque sea de "manera virtual" (Leibniz, 1704/1992, 74) o de "modo implícito" (Leibniz, 1704/1992, 83). Propiamente hablando, lo que existe es una facultad cognoscitiva innata, capaz de generar autosuficientemente ideas que, atendiendo a su origen, llamamos a su vez también innatas.

Lo cierto es que el innatismo disposicional, aunque no haya sido esta obviamente la voluntad de Descartes, apunta a una inevitable disolución de cualquier sentido claro en asociación con el concepto de lo innato. El disposicionalismo cartesiano y la deriva naturalista a que parece inevitablemente dar lugar pueden resumirse así. Lo que resulta innato a la mente es un tipo de disposición, comparable a otras tantas asimismo patentes en el organismo humano, que en último termino remite a un generador de ideas capaz de actuar incluso en ausencia de estímulos o modelos ambientales. La comparación con lo orgánico, por su parte, da lugar a que el generador en cuestión sea conceptualizable como un mecanismo de ideación y, como tal, potencialmente sujeto al mismo tipo de explicación mecanicista que los demás órganos que igualmente amparan disposiciones. Por tanto, también al igual que cualquiera de estos, el desarrollo del mecanismo generativo en cuestión sería susceptible de recibir el mismo tipo de explicación mecánica característica de la visión epigeneticista apoyada por el propio Descartes, basada en la concatenación de procesos e interacciones contingentes entre materiales internos y externos al organismo. Cualquier sentido claro del concepto de innatismo se pierde en esta deriva.

En el fondo, como razonó con especial claridad Jerry Fodor en diferentes trabajos, si lo innato no son las ideas, sino los mecanismos, entonces tan innatistas son, digamos, Descartes como Locke (Fodor, 1998; 2003). La diferencia es, como mucho, cuantitativa, pero en absoluto cualitativa. El innatismo disposicional apunta, pues, a la disolución del debate clásico entre los defensores de la experiencia y los defensores de la razón como proveedores principales del caudal cognoscitivo humano, o lo deja, en todo caso, en una especie de tablas. Para lo que aquí interesa, el sentido de "lo innato" se pierde con la disolución de la contraposición tradicional, fuera de la cual se vacía de toda semántica. De ahí que Lorenzo y Longa escriban que "la secular polémica en torno a la idea de innatismo puede tener algo de malentendido; ciertamente, un largo y nunca debidamente aclarado malentendido" (Lorenzo y Longa, 2018, 193; véase, también, Savile, 1972, 121). Patente demostración de ello es que, al abrigo de 
un innatismo disposicional y exclusivamente referido a los mecanismos, pueda hablarse, como bien razonó Fodor (2003), de un "cartesianismo naturalista" en el que tendrían cabida por igual el Leibniz disposicionalista y el Hume empirista.

La caída de las ideas innatas tal vez habría sido una pérdida históricamente asumible por el partido racionalista. Ahora bien, cuestión bien diferente es que la naturalización del cartesianismo se llevase además por delante su idea de "mente", al subsumirla en el mismo plano explicativo que la sustancia extensa. La naturalización de la mente rompería definitivamente las tablas del debate en favor de un empirismo cientificista. En la clarificación de la idea de "naturalismo" que Jerry Fodor y Zenon Pylyshyn aportan en el mapa conceptual con que abren su muy relevante Mind without meanings queda particularmente claro que, en tal marco, resulta imposible preservar a la idea de "mente" cualquier tipo de autonomía frente a lo empírico:

Los estados y los procesos mentales son parte del mundo físico. Esto significa, como poco, que los procesos que la ciencia cognitiva postula deben ser realizados por mecanismos físicos reales, y que los estados que asimismo postula deben ser tales que los objetos físicos puedan manifestarlos (Fodor y Pylyshyn, 2015, 3-4; trad. de GL).

Es posible leer la Monadología de Leibniz como un poema filosófico, casi a la manera de los textos presocráticos. Con esta afirmación, o sugerencia, intento subrayar las cualidades formales del tratado, que se presta a ser explorado por el puro placer de la exploración, sin llegar más que a vislumbrar un difuso sentido. Filosóficamente, en el contexto trazado por los párrafos precedentes, la Monadología asegura a la mente cualidades (autoconsistencia, indisolubilidad, indestructibilidad...) capaces de mantenerla lejos del peligro de la naturalización a que la aboca un innatismo disposicional. En otras palabras, la apuesta definitiva de Leibniz, parafraseando a Fodor, aunque a la inversa, por un "cartesianismo antinaturalista”.

La propiedad clave de la mente leibniziana, como ya había sido con anterioridad del alma platónica (Fedón, 78c3) o sería, más tarde, de los conceptos fodorianos (Fodor, 1998), es la de ser una sustancia indisoluble o inarticulada. 
Es esta propiedad la que la separa de raíz del mundo físico, pues la indivisibilidad de lo mental garantiza que el tipo de principio reactivo en que se basan las respuestas de la mente a los cambios del mundo físico (comenzando por los del propio cuerpo) no se corresponde con el tipo de causalidad mecánica propio de estos, basado en el efecto del impacto causal entre partes. Como señala Alberto Relancio, el marco mecanicista servía a Leibniz como una "garantía de racionalidad en el nivel de los fenómenos físicos” (Relancio Menéndez, 2009, 157). El abismo entre lo físico y lo mental se abre, pues, en el momento en que la causalidad mecánica se detiene ante la mente, cuya capacidad definitoria es la de registrar o "representar" cualquier cambio en el mundo físico, con intensidad proporcionada a su proximidad a los cambios en cuestión, como una leve modificación en la representación que le es a priori propia de la totalidad del universo. Se sigue, pues, un innatismo generalizado de las representaciones mentales.

Del planteamiento innatista generalizado se sigue, de modo tajante, que el estudio de la mente y sus potencias (su capacidad de registrar cambios o "percibir", de sentir inclinaciones o "apetencias" hacia ellos y de actuar "voluntariamente" en consecuencia) escapan al tipo de comprensión que brinda la de los cuerpos concebidos como mecanismos. Ciertamente, la cuestión es más compleja, pues, por un lado, Leibniz "no renuncia al mecanicismo en el campo de los fenómenos biológicos" (Relancio Menéndez, 2009, 168), aunque, por otro lado, atribuye a los constituyentes orgánicos de los cuerpos físicos cualidades parcialmente equiparables a los de la mónada mental (unidad, indisolubilidad, incorruptibilidad, comunicación armónica con el resto de las mónadas). En último término, con palabras también tomadas de Relancio Menéndez, la de Leibniz acaba por ser una "física ficción" (Relancio Menéndez, 2009, 171), enmarcada en una metafísica generalizada en que la frontera entre lo físico y lo mental, entre el mecanismo y la representación, acaba por borrarse en favor de los últimos elementos de las dicotomías de partida.

La implicación de Leibniz en los más diversos proyectos de investigación y difusión científicos está fuera de cualquier duda (Rada, 2002). Algunos autores han defendido que Leibniz no quiso dejar su Monadología totalmente desprovista de una cierta verosimilitud científica (Relancio Menéndez, 2009; Russo, 1931; Smith, 2002a; Wilson, 1997); y, a la inversa, que la visión metafísica resultante alentó ciertas visiones crecientemente pujantes en la ciencia coetánea o subsiguiente (Rieppel, 1988; Smith, 2002b). Maienschein (2012) destaca que en tiempos de Leibniz el preformacionismo comenzó a imponerse sobre el epigeneticismo, anteriormente dominante, por motivos empíricos (la interpretación 
de nuevas observaciones microscópicas) y epistemológicos (la resistencia a aceptar la inteligibilidad de la conformación a partir de lo informe). Leibniz pudo beneficiarse de esta pujante weltanschauung y también retribuirla con una cierta coartada metafísica. Es decir, como razona acertadamente Justin E. Smith, "Leibniz se sirvió de los hallazgos de la ciencia empírica para sentirse seguro al anunciar sus principios metafísicos a la intelectualidad" (Smith 2002a: 162), "que de otro modo habrían parecido bastante extravagantes" (Smith 2002a: 179); sin embargo, esos principios "eran independientes, en su desarrollo y contenido, a los descubrimientos de la ciencia natural de la época" (Smith 2002a: 166; trads. de GL).

Todo lo anterior significa que el innatismo tiene un encaje mucho más coherente con una concepción preformacionista de lo orgánico que con la concepción alternativa epigeneticista. Una de las ideas que he sostenido en este texto es la de que la interpretación disposicionalista del innatismo, defendido por Descartes e inicialmente por Leibniz, y, más aún, su posible encaje con la visión epigeneticista del desarrollo orgánico de la que participó además el primero, eran proyectos autodestructivos para la posición innatista. Probablemente, su primer naufragio histórico, anticipando todos los que le seguirían ya en el siglo XX. Al contrario, en la isla metafísica de Leibniz el innatismo se rearma sin complejos, reafirmándose como una tesis en que la mente coexiste en armonía con los cuerpos físicos, sin confundirse con estos. Rotas todas las amarras con cualquier proyecto científico proclive a la naturalización de la mente, la insularidad monadológica no parece que fuera tanto el precio que Leibniz había de pagar por preservar la primacía de lo mental, como un honroso distintivo de su triunfo.

\section{Bibliografía}

Aucante, Vincent (2006). "Descartes' experimental method and the generation of animals". Ed. Smith, J.E., The problem of animal generation in early modern philosophy. Cambridge: Cambridge University Press, pp. 65-79.

Balari, Sergio, Longa, Víctor M., y Lorenzo, Guillermo (2020). "Nothing makes sense in grammar, except in the light of development". Ms. Universitat Autònoma de Barcelona, Universidade de Santiago de Compostela y Universidad de Oviedo.

—, Lorenzo, Guillermo y Sultan, Sonia (2020)."Language acquisition and ecodevo processes: the case of the lexicon-syntax". Biological Theory (online first, https://doi. org/10.1007/s13752-020-00352-9). 
Berwick, Robert, Pietroski, Paul, Yankama, Beracah y Chomsky, Noam (2011). "Poverty of stimulus revisited". Cognitive Science Vol. 35, pp. 1207-1242.

Сномкку, Noam (1959). "A review of B.F. Skinner's Verbal Behavior". Language Vol. 35, pp. 26-58.

- (1967). "Recent contributions to the theory of innate ideas". Synthese Vol. 17, pp. 2-11.

- (1980). Rules and representations. New York: Columbia University Press.

- (2005). "Three factor in language design". Linguistic Inquiry Vol. 36, pp. 1-22.

- (2009). "Opening remarks". Eds. Piatelli-Palmarini, M., Uriagereka, J. y Salaburu, P., Of minds and language. A dialogue with Noam Chomsky in the Basque Country. Oxford: Oxford University Press, pp. 13-43.

- (2012). The science of language. Interviews with James McGilvray. Cambridge: Cambridge University Press.

- (2016). "Poverty of stimulus: unfinished bussines". Studies in Chinese Linguistics Vol. 33, pp. 3-16.

Cowie, Fiona (1999). What's within. Nativism reconsidered. Oxford: Oxford University Press.

Descartes, René (1637/1987). Du foetus (Ed. Pardos, P., Vicén, C. y Alonso, A.). Zaragoza: Prensas Universitarias.

- (1648/1981). Observaciones sobre la explicación de la mente humana (Ed. Quintás, G.). Madrid: Teorema.

FoDor, Jerry A. (1998). Concepts. Where cognitive science went wrong. Cambridge, MA: The MIT Press.

- (2003). Hume variations. Oxford: Oxford University Press.

Gorham, Geoffrey (2002). "Descartes on the inateness of all ideas". Canadian Journal of Philosophy Vol. 32, pp. 355-388.

GоттLіев, Gilbert (1997). Synthesizing nature-nurture: prenatal roots of instinctive behavior. Mahwah, NJ: Lawrence Erlbaum.

Griffiths, Paul y Stotz, Karola (1997). Genetics and philosophy. An introduction. Cambridge: Cambridge University Press.

KIm, Halla (2003). "Locke on innatism”. Locke Studies Vol. 3, pp. 15-39.

Kuo, Zing Yang (1921). "Giving up instincts in psychology". The Journal of Philosophy Vol. 18, pp. 645-664.

— (1922). "How are our instinct acquired". The Psychological Review Vol. 29, pp. 344365.

- (1924). "A psychology without heredity". The Psychological Review Vol. 31, pp. 427448. 
- (1932). "Ontogeny of embryonic behavior in aves: I. The chronology and general nature oft he behavior oft he chick embryo". Journal of Experimental Zoology Vol. 61, pp. 395-430.

- (1967). The dynamics of behavior development. Nueva York: Plenum Press.

Lehrman, Daniel S. (1921). "A critique of Konrad Lorenz's theory of instinctive behavior". The Quarterly Review of Biology Vol. 28, pp. 337-363.

Leibniz, Gottfried W. (1704/1992). Nuevos ensayos sobre el entendimiento humano (Ed. Echevarría Ezponda, J.). Madrid: Alianza.

LocKE, John (1690/2013). Ensayo sobre el entendimiento humano (Ed. O'Gorman, E.). México: Fondo de Cultura Económica.

LockE, John (1983). The child's path to spoken language. Cambridge, MA: Harvard University Press.

Longa, Víctor M. (2008). "Una visión crítica sobre la noción de 'programa genético' desde la biología y la lingüística: consecuencias para la conceptualización de la ontogenia del lenguaje". Verba. Anuario Galego de Filoloxía Vol. 35, pp. 347-385.

— (2018). "That was not Lenneberg's dream”. Historiographia Linguistica Vol. 45, pp. 179-209.

- y Lorenzo, Guillermo (2012). "Theoretical linguistics meets development: explain FL from an epigeneticist point of view". Eds. Boeckx, C., Horno-Chéliz, M.C. y Mendívil-Giró, J.L., Language, from a biological point of view. Current issues in biolinguistics. Newcastle upon Tyne: Cambridge Scholars Publishing, pp. 52-84.

LoRenzo, Guillermo (2001). Comprender a Chomsky. Introducción y comentarios a la filosofia chomskiana sobre el lenguaje y la mente. Madrid: Antonio Machado Libros.

- y Longa, Víctor M. (2018). El innatismo. Orígenes, variaciones y vitalidad de una idea. Madrid: Cátedra.

Maienschein, Jane (2012). "Epigenesis and preformationism". Ed. Zalta, E.N., The Stanford Encyclopedia of Philosophy (edición de primavera de 2012), URL $=<$ http.// plato.stanford.edu/archives/spr2012/entries/egigenesis>

Mehler, Jacques y Dupoux, Emmanuel (1990). Naître humain. Paris: Odile Jacob.

PeÑA, Vidal (2005). "Introducción”. En Descartes, R. Meditaciones metafísicas con objeciones y respuestas (Ed. Peña, V.). Oviedo: KRK.

Rada, Eloy (2002). "Leibniz y la ciencia”. Themata Vol. 29, pp. 141-150.

Rawson, Glenn (2006). "Platonic recollection and mental pegnancy". Journal oft he History of Philosophy Vol. 44, pp. 135-155.

Relancio Menéndez, Alberto (2002). "La influencia de la biología en la Monadología de Leibniz". Themata Vol. 42, pp. 155-182.

Richards, Robert J. (1987). Darwin and the emergence of evolutionary theories of mind and behavior. Chicago: The University of Chicago Press. 
RIEPPEL, Oliver (1988). "The reception of Leibniz's philosophy in the writings of Charles Bonnet (1720-1793)". Journal of the History of Philosophy Vol. 21, pp. 119145.

Russo, Salvatore (1931). "The influence of the theory of preformation on Leibniz's metaphysics". The Open Court Vol. XLV, pp. 257-267.

SAMET, Jerry (2008). "The historical controversies surrounding innateness". Ed. Zalta, E.N., The Stanford Encyclopedia of Philosophy (edición de otońo de 2008), URL = $<$ http.//plato.stanford.edu/archives/fall2008/entries/innateness-history>

Savile, Anthony (1972). "Leibniz's contribution to the theory of innate ideas". Philosophy Vol. 47, pp. 113-124.

SMIT, Harry (2020). "The Cartesian conception of the development of the mind and its neo-Aristotelian alternative”. Biological Theory Vol. 15, pp. 107-120.

Sмiтh, Justin Erik (2002a). "Leibniz's preformationism: between metaphysics and biology". Analecta Husserliana: The Yearbook of Phenomenological Research Vol. 77, pp. 161-192.

- (2002b). "Leibniz's hylomorphic monad". History of Philosophy Quaterly Vol. 19, pp. 21-42.

Sтісн, Stephen P. (1975). “The idea of innateness". Ed. Stich, S.P, Innate ideas. Berkeley y Londres: University of California Press (reimpreso en Stich, S.P. Collected papers. Vol. I. Mind and language, 1972-2010. Oxford, Oxford University Press, 2011, pp. 20-35)

Wilson, Catherine (2012). "Leibniz and the Animalcula". Ed. Stewart, M.A., Studies in the Seventeenth-Century European Philosophy. Oxford: Oxford University Press, pp. 153-176

Recibido: 7/10/2020

Aceptado: 20/11/2020

Este trabajo se encuentra bajo una licencia de Creative Commons Reconocimiento-

NoComercial-SinObraDerivada 4.0 
